\title{
Patterns of care study of brachytherapy in New South Wales: cervical cancer treatment quality depends on caseload
}

\author{
Dr. Stephen R. Thompson, MBBS, PhD, FRAnZCR',2,3, Prof. Geoff P. Delaney, MBBS, MD, PhD, FrAnZCR',3,4, \\ Dr. Gabriel S. Gabriel, MBBS, MPH, FAFPHM!'3, Prof. Michael B. Barton, OAM, MBBS, MD, FRANZCR,3 \\ 'Collaboration for Cancer Outcomes Research and Evaluation (CCORE), Liverpool Hospital, Sydney, ${ }^{2}$ Department of Radiation Oncology. \\ Prince of Wales Hospital, Sydney, ${ }^{3}$ University of New South Wales, Sydney, ${ }^{4}$ University of Western Sydney, Sydney, NSW, Australia
}

\begin{abstract}
Purpose: We previously conducted modelling and a patterns of care study (POCS) that showed gynaecological brachytherapy (BT) was underutilized in New South Wales (NSW), the USA and Western Europe. The aim of the current study was to assess the quality of cervical BT in NSW, and to determine if caseload affects quality of treatment delivery.

Material and methods: All nine NSW radiation oncology departments that treated patients with cervical BT in 2003 were visited. Patient, tumour and treatment related data were collected. Quality of BT was assessed using published quality benchmarks. Higher and lower caseload departments were compared.

Results: The four higher cervical BT caseload departments treated 11-15 NSW residents in 2003, compared to 1-8 patients for the lower caseload departments. Cervix cancer patients treated at the higher caseload departments were more likely to be treated to a point A dose $\geq 80$ Gy ( $58 \%$ vs. $14 \%, p=0.001)$, and to have treatment completed within 8 weeks $(66 \%$ vs. $35 \%, p=0.02)$. Despite higher point A doses, there was no significant difference in proportions achieving lower than recommended rectal or bladder doses, implying better BT insertions in higher caseload departments.

Conclusions: Cervical BT in NSW was dispersed amongst a large number of departments and was frequently of sub-optimal quality. Higher quality BT was achieved in departments treating at least 10 patients per year. It is likely that improved outcomes will be achievable if at least 10 patients are treated per department per year.
\end{abstract}

J Contemp Brachytherapy 2014; 6, 1: 28-32

DOI: $10.5114 / j c b .2014 .42022$

Key words: brachytherapy, caseload volume, cervical carcinoma, patterns of care studies, quality, radiotherapy.

\section{Purpose}

We performed the first comprehensive, populationbased brachytherapy (BT) patterns of care study (POCS) in the Australian setting by performing a BT POCS for New South Wales (NSW). In comparison with our model of optimal BT utilization, gynaecological BT was underutilized in NSW, as well as in the USA and Western Europe [1]. The other aim of the POCS was to assess the technical quality of NSW BT practice by comparing with known quality benchmarks, and to thereby determine if a caseload effect exists. Herein we focus on these results as they pertain to cervical BT quality. Although uncommon in Australia, worldwide cervical carcinoma is the fourth largest cause of cancer death in females [2]. Brachytherapy is an integral part of cervical cancer treatment [3], and the technical quality of cervical BT has been shown to cor- relate with outcome [4]. Previous cervical cancer POCS have shown that institution size/type affects cervical treatment quality and outcome [5,6], but as yet there is no data directly comparing institutional cervical BT caseload and cervical BT quality.

\section{Material and methods}

In 2005, a retrospective NSW BT POCS was performed for patients treated in 2003. The population for the current study was all NSW resident cervical cancer patients treated with BT in NSW in 2003. Site visits and data collection by the principal investigator were made to all 9 radiation oncology departments in NSW that deliver BT. Collected data were entered into an Access database and then transferred into SPSS v15.0. Logic checks and frequency analyses were performed to identify data errors and in- 
consistencies. Quality of BT treatment was assessed using published benchmarks: point A dose $\geq 80$ Gy [3,7], ICRU (International Commission on Radiation Units and Measurements) rectal point dose < 75 Gy [3], ICRU bladder point dose $<80$ Gy [3], and total treatment time $\leq 8$ weeks $[3,7]$. Quality was compared between the four departments that performed more cervical BT (higher BT caseload) compared to the five departments that performed fewer (lower BT caseload). Doses were compared using the linear quadratic equation to convert high dose rate (HDR) BT doses to equivalent low dose rate (LDR) doses, which are approximately equivalent to standard fractionation at 2 Gy per fraction (EQD). For late effects, $\alpha / \beta=3$, and for early effects and modelling of tumour control $\alpha / \beta=10$ [8].

Categorical variables were compared using the $\chi^{2}$ test. Means of two variables were compared using the two-sample $t$-test (two-tailed). Pearson correlation test was performed to assess relationships between bladder/rectal doses and point A doses. All institutions and patients were de-identified. Permission for the study was obtained from all relevant Radiation Oncology Departmental Directors and Human Research Ethics Committees. The study was carried out in accordance with the Helsinki Declaration.

\section{Results}

In NSW in 2003, there were 15 radiation oncology departments, of which $9(60 \%)$ treated patients with BT, all administering gynaecological BT. After exclusion of 14 cervical cancer patients resident interstate, 76 NSW cervical cancer patients were treated with BT in 2003. The median number of cases per department was 8 . Four departments treated 52 (68\%) patients, median 13 (range 11-15) per department, and are categorized in further analysis as "higher cervical BT caseload departments". The other five departments treated the remaining 24 (32\%) patients, median 4 (range 1-8) per department, and are categorized as "lower cervical BT caseload departments".

Mean patient age was 59 (range 26-83). Fifty-seven (75\%) patients were stage IIB-IVA, with technically inoperable disease. Seventy-five patients had known histology: with most $(87 \%)$ having squamous cell carcinomas, the remainder being adenocarcinomas $(9 \%)$ or adenosquamous carcinomas $(4 \%)$. One patient was treated for locally recurrent disease, and five patients were treated adjuvantly. Definitive radiotherapy with EBRT and BT for 70 patients with non-recurrent disease forms the subject of the remainder of the analysis.

Tandem and ovoid BT was the commonest applicator type, used for $61(87 \%)$ patients, with 6 of 9 departments using only this equipment. LDR ${ }^{137} \mathrm{Cs}$ BT was used by three departments to treat $40(57 \%)$ patients, PDR ${ }^{192} \mathrm{Ir}$ was used by two departments (treating as LDR) to treat $5(7 \%)$ patients, and HDR ${ }^{192}$ Ir BT was used by four departments treating $25(36 \%)$ patients. All departments used orthogonal film based planning. Seven departments used individualized planning, while two (both lower caseload departments) applied a best-fit library plan to their nine anaesthetised HDR patients. All departments dosed to point A. There was no difference in type of ap- plicator used by caseload, with almost all centres using tandem and ovoids. Lower caseload departments were more likely to treat using a best-fit library (rather than individualised) plan, treating $38 \%$ versus $2 \%$ of patients this way ( $p=0.002$, OR: $0.034,95 \%$ CI: 0.004-0.296). Dose data for EBRT and BT were available for 69 of the 70 patients. No consistent dosing schedule was used, with a wide range of doses and fractionations prescribed across the state. Median EBRT dose to the cervix was 50 Gy (range: 39-50.4 Gy) in 1.8 Gy or 2 Gy fractions for all except two patients who were treated with $1.67 \mathrm{~Gy}$ fractions. Median and modal HDR dose was 18 Gy (range: 6-24 Gy), delivered in one ( $8 \%$ of patients), two $(8 \%)$, or usually three fractions (84\%). Dose was known for 44 of the 45 patients treated in an LDR fashion (with LDR or PDR equipment): median and modal dose was 30 Gy (range: 20-43 Gy), in one $(69 \%$ of patients), two $(29 \%)$, or rarely three fractions $(2 \%)$. Median total EQD2 dose to point $A(\alpha / \beta=10)$ was 76 Gy (range: 47-88 Gy). Higher caseload departments treated to higher mean doses (78 Gy vs. $71 \mathrm{~Gy}, p<0.001,95 \% \mathrm{CI}$ for difference: 3.5-10.2 Gy). Benchmark dose $\geq 80 \mathrm{~Gy}$ was delivered in only $31(45 \%)$ patients with higher caseload departments more likely to achieve this benchmark: 28 of $48(58 \%)$ compared with 3 of $21(14 \%)(p=0.001$, OR: 8.40, 95\% CI: 2.18-32.4) (Fig. 1).

HDR patients were treated to lower doses than LDR patients (mean 72 Gy vs. 79 Gy, $p<0.001,95 \%$ CI for difference: 3.5-9.8 Gy), and only 5 of 25 (20\%) HDR patients were treated to the benchmark point A dose, compared to 26 of $44(59 \%)$ LDR patients ( $p=0.002$, OR: $0.17,95 \%$ CI: $0.05-0.55)$. When using HDR BT, higher caseload departments were more likely to treat to an adequate dose (4 of 9 patients, $44 \%$ ) compared to lower caseload departments ( 1 of 16 patients, $6 \%$ ) ( $p=0.02$, OR: $12.0,95 \%$ CI: $1.07-134$ ). This distinction was not necessarily true in the case of LDR BT: adequate dose was achieved for 24 of 39 (62\%) patients treated in higher caseload departments compared to 2 of $5(40 \%)$ patients treated in lower caseload departments, but this difference was not statistically significant.

ICRU rectal point data were routinely recorded by all departments and were available for 66 of the 70 (94\%) pa-

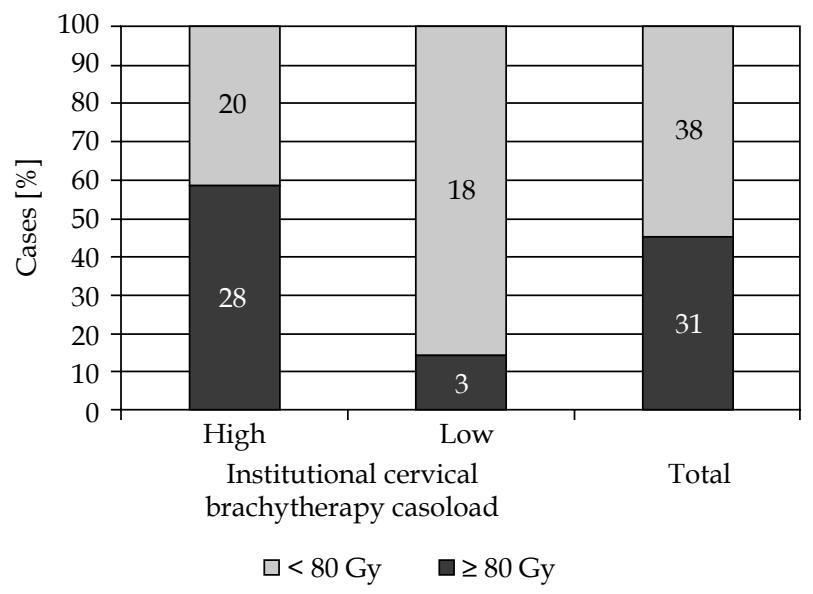

Fig. 1. Adequacy of point A dose, by institutional cervical brachytherapy caseload $(p=0.001)$. Adequate dose is EQD2 $(\alpha / \beta=10) \geq 80 \mathrm{~Gy}$ 


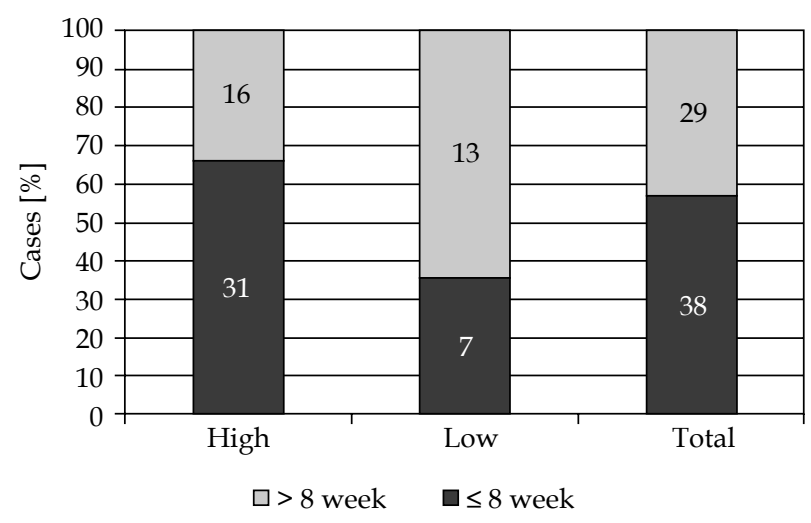

Fig. 2. Treatment duration, by institutional cervical brachytherapy caseload $(p=0.019)$. Adequate treatment duration is less than 8 weeks

tients. Median total EQD2 dose to the ICRU rectal point $(\alpha / \beta=3)$ was 64 Gy (range: $43-77 \mathrm{~Gy})$. There was no statistically significant difference in mean doses by departmental caseload $(p=0.11)$. The recommended dose of $<75 \mathrm{~Gy}$ was achieved in 61 of $66(92 \%)$ patients with no differences by caseload $(p=0.6)$. Although HDR patients averaged lower rectal doses (HDR 60 Gy vs. LDR 66 Gy, $p<0.001$, 95\% CI for difference: 3.8-9.8 Gy), there was no significant difference in the likelihood of the < 75 Gy benchmark being achieved by BT dose rate (HDR $96 \%$ of cases vs. LDR $91 \%, p=0.47$ ). Point A doses and rectal doses were weakly correlated $(r=0.449)$. ICRU bladder point dose data were routinely recorded by all departments except one, and were available for 56 of the $70(80 \%)$ patients. Median total EQD2 dose to the ICRU bladder point $(\alpha / \beta=3)$ was 80 Gy (range: 53-175 Gy). There was no significant difference in mean doses by departmental caseload ( $p=0.23$ ). The benchmark dose $<80$ Gy was achieved in 28 of 56 $(50 \%)$ patients with no differences by caseload $(p=0.34)$. HDR patients averaged higher bladder doses (HDR 94 Gy vs. LDR $77 \mathrm{~Gy}, p=0.04$, 95\% CI for difference: 1.3-32 Gy) and HDR BT was significantly less likely to achieve a bladder point EQD2 dose less than the recommended $80 \mathrm{~Gy}$ maximum (HDR $29 \%$ of cases vs. LDR 59\%, $p=0.042$, OR: 0.29, 95\% CI: 0.09-0.98). There was no correlation between point $\mathrm{A}$ doses and bladder doses.

Total treatment time was calculable for all but 3 patients (96\%). Median treatment duration was 54 days (range 37-75 days). A maximum of 8 weeks is recommended after which accelerated repopulation may negatively affect survival $[3,7]$. This benchmark was achieved in the treatment of 38 of $67(57 \%)$ patients, more commonly in higher caseload departments (31 of 47 patients, $66 \%$ ), compared to lower caseload (7 of 20 patients, 35\%) $(p=0.02$, OR: 3.60, 95\% CI: 1.20-10.8) (Fig. 2). There was no statistically significant difference between HDR or LDR treatments $(p=0.27)$.

\section{Discussion}

In NSW in 2003, 76 NSW residents with cervical cancer underwent BT, in 70 of whom treatment was part of definitive radiotherapy. At this time, no NSW radiotherapy departments were using 3-dimensional BT treatment plan- ning, the majority of patients were treated with LDR BT, and many patients were treated to inadequate total XRT doses over prolonged treatment time, particularly in the departments that treated fewer patients with cervical BT.

Comparing the results of our NSW POCS with the Australasian POCS performed only two years later by van Dyk et al. shows that there appears to be a move towards incorporation of more advanced BT technology [9]. Only two years later, 6 of 21 (29\%) Australasian departments had incorporated some form of 3-dimensional treatment planning [9]. This has been shown to improve tumour control and treatment related toxicity [10]. There is also a move towards HDR BT, which was used in NSW in 2003 by 4 of the $9(44 \%)$ departments for 25 of the $70(36 \%)$ definitively treated patients. Two years later, according to van Dyk's survey, 12 of 21 (57\%) Australasian departments were using HDR to treat cervical cancer, reflecting the gradual replacement of LDR by HDR for the treatment of cervical cancer as manufacture of this equipment wound down [9]. Given this move to HDR BT, a concern is that NSW benchmark point A doses for HDR BT compared to LDR were less likely to be reached (20\% vs. $59 \%$, $p=0.002$ ), and benchmark bladder doses were also less likely to be achieved ( $29 \%$ vs. $59 \%, p=0.042)$. A reason for this poor therapeutic ratio may be the use of insufficient fractionation in NSW, with HDR BT usually delivered in three fractions of $8 \mathrm{~Gy}$. This compares unfavourably with the Japanese and Canadian medians of four HDR fractions $[11,12]$, and the US median of 5-6 [13]. Fewer fraction numbers implies increased HDR BT fraction size for a given total dose and is correlated with increased late toxicity [14].

For optimal local control, guidelines recommended total point A doses greater than EQD2 80-85 Gy [3,7]. In NSW, this benchmark was achieved in only $45 \%$ of patients. This compares unfavourably with the 1996-1999 POCS in the USA, showing that $69 \%$ of patients were treated to a point A dose of $\geq 80$ Gy [5]. In NSW, median total EQD2 dose to point A was 76 Gy for the entire cohort (72 Gy for HDR BT and 79 Gy for LDR BT). By comparison, overseas doses were higher. In a Canadian survey, median prescribed EQD2 for HDR BT was 83 Gy [12], and in the 1996-1999 USA POCS, median total EQD2 for HDR BT was 86 Gy and 83 Gy for LDR BT [13]. Japanese doses were lower, with median EQD2 of only 62 Gy [11]. It is likely that local control may be sub-optimal in NSW patients as a result of inadequate point $\mathrm{A}$ doses.

Bowel and bladder complications of definitive radiotherapy for cervical cancer are not infrequent, can be severe, and severity and frequency correlate with dose; guidelines therefore recommend routine calculation of doses to organs at risk [3]. New South Wales departments demonstrated a high rate of compliance with the documentation required, with all departments recording rectal doses and 8/9 (88\%) recording bladder doses, similar to Canada (89\% rectal doses and $86 \%$ bladder doses) and comparing favourably to Japan (25\% and $18 \%)$ and USA (25\% overall for HDR and 93\% for LDR) [11-13]. The New South Wales POCS showed that $92 \%$ of patients were treated to a rectal point dose of less than the recommend- 
Table 1. New South Wales cervical brachytherapy caseload per centre and comparison with other jurisdictions

\begin{tabular}{|c|c|c|c|c|c|c|}
\hline \multirow{3}{*}{$\begin{array}{l}\text { Cases/Centre/ } \\
\text { Year }\end{array}$} & \multicolumn{3}{|c|}{ NSW } & \multirow{3}{*}{$\begin{array}{c}\text { ABS } \\
1995^{\star} \\
{[16]}\end{array}$} & \multirow{3}{*}{$\begin{array}{c}\text { US POCS } \\
1996-99 \\
{[13]}\end{array}$} & \multirow{3}{*}{$\begin{array}{c}\text { UK } \\
2004 \\
{[17]}\end{array}$} \\
\hline & & 2003 & & & & \\
\hline & All & Higher caseload & Lower caseload & & & \\
\hline Mean & 8.4 & 13.0 & 4.8 & - & 7.4 & - \\
\hline Median & 8 & 13 & 4 & 6.4 & - & 19 \\
\hline Range & $1-15$ & $11-15$ & $1-8$ & - & - & $9-45$ \\
\hline$>10$ cases & $44 \%$ & $100 \%$ & $0 \%$ & $25 \% \geq 15$ & $40 \% \leq 2$ & not stated \\
\hline
\end{tabular}

NSW - New South Wales, ABS - American Brachytherapy Society, US POCS - United States Patterns of Care Study, UK - United Kingdom, - not available; * ABS 1995 data per Radiation Oncologist

ed maximum of $75 \mathrm{~Gy}$ [3], but only $50 \%$ of patients were treated to a bladder point dose of less than the recommended maximum of 80 Gy [3]. The greater likelihood of patients being treated within rectal tolerance rather than bladder tolerance probably reflects the known greater risk of gastrointestinal toxicity compared to bladder toxicity after definitive radiotherapy for cervical cancer [15].

Given accelerated repopulation of tumour clonogens in cervical cancer, guidelines recommend treatment completion within 8 weeks [3,7]. This benchmark was achieved in only $57 \%$ of cases, with local control likely to be adversely impacted as a result. This compares favourably with the $47 \%$ rate in the 1996-1999 USA POCS [13], but unfavourably with the $72 \%$ rate reported by the Japanese 1999-2001 POCS [11].

Technical quality of brachytherapy insertion for cervical cancer has been correlated with local control and toxicity with a strong trend towards improved survival $[4,16]$. Given this, an important issue is whether or not cervical brachytherapy caseload affects quality. The American Brachytherapy Society (ABS) has recommended that radiation oncologists with low cervix cancer caseloads (less than four cases per year) refer to larger centres or more experienced physicians [17]. In the UK in 2007 it has been recommended that a minimum of 10 cases be treated per centre per year, although no data were referenced to support this recommendation [18]. Our results support these ABS and UK assertions by showing a direct correlation between departmental cervical BT caseload and BT quality. The higher caseload departments were more likely than lower caseload departments to complete treatment within the benchmark 8 weeks (66\% vs. $35 \%$ of cases, $p=0.02$, Fig. 2), to treat to the recommended point A dose $\geq 80$ Gy ( $58 \%$ vs. $14 \%$ of cases, $p=0.001$, Fig. 1 ), and to treat using an individualised rather than library plan. As there were no significant differences between higher and lower caseload departments in mean rectal and bladder point doses (or in the proportions of patients with rectal or bladder doses greater than recommended), the higher prescribed point A doses of the higher caseload departments imply that these higher doses were achieved by technically superior BT applicator placement, given that there was no cost in terms of higher doses to organs at risk. The NSW results are consistent with the international literature, generally (but not always) showing significant correlations between departmental caseload or facility size/type and treatment quality/outcomes [5,6,19-21].
However, our NSW POCS data are the first data to directly compare cervical BT quality benchmarks by institutional cervical BT caseload. All the higher cervical BT workload NSW departments treated more than 10 cases per year, lending support to the UK Royal College of Radiologists' assertion that the minimum number of cases per centre per year should be ten [18].

Considering that in NSW 5 of 9 departments did not meet this benchmark, that patients treated in these departments were significantly more likely to be treated to lower doses over a longer treatment time with (probably) sub-optimal implants, and that the median number of cervical BT cases per centre in NSW is less than half that of the UK (see Table 1), there is an implication that cervical cancer outcomes in NSW may be improved if cervical BT were to be centralised. The same conclusions might be drawn from the data showing low caseload per centre in the USA (Table 1). Given that we have already shown that gynaecological BT is underutilized in NSW and the USA [1], this would have significant implications for health resource planning and patterns of patient care and referral.

This study did have a number of limitations. It was retrospective, involved small patient numbers, and represents state-of-play in 2003, and therefore not necessarily reflecting current NSW practice. Nevertheless, our results remain applicable: in the UK in 2012, dose was prescribed to a volume in only $29 \%$ of centres [22]. In the developing world, many centres, treating the majority of the world's cervical cancer patients [2], are probably using 2-dimensional treatment planning and dosing to point A. As cervical BT becomes more conformal with the adoption of MRI-based volumetric planning, although target definition is safe in expert centres [23], there may be a greater risk of geographical miss and poorer tumour control in inexperienced hands. The skills required to perform an adequate BT insertion remain unchanged. Importantly, this study reports technical factors, but not outcome data. The lower point A doses and longer treatment times in patients treated at lower caseload departments are likely to have resulted in poorer disease control $[24,25]$. Assuming, the present results are valid, it is not known whether or not further improvements in BT quality might be achieved by increasing the recommended minimum number of cases per centre further beyond ten. These are areas in which future research is likely to be beneficial. 


\section{Conclusions}

Cervical BT in NSW in 2003 was dispersed amongst a large number of departments and was of sub-optimal quality in the lower caseload departments. Higher quality brachytherapy was achieved in departments treating at least 10 patients per year. Although previous studies have shown improved treatment quality in larger and/ or academic radiation oncology departments, this is the first study showing an association between cervical BT caseload and treatment quality, and it is likely that improved outcomes will be achievable if at least 10 patients are treated per department per year.

\section{Acknowledgements}

The authors thank the Directors of Radiation Oncology Departments in New South Wales who provided access to patient data.

\section{Disclosure}

Authors report no conflict of interest.

\section{References}

1. Thompson SR, Delaney GP, Gabriel SG et al. Estimation of the optimal brachytherapy utilization rate in the treatment of gynaecological cancers and comparison with patterns of care. Int J Radiat Oncol Biol Phys 2013; 85: 400-405.

2. Ferlay J, Shin HR, Bray F et al. Estimates of worldwide burden of cancer in 2008: GLOBOCAN 2008. Int J Cancer 2010; 127: 2893-2917.

3. Nag S, Chao C, Erickson B et al. The American Brachytherapy Society recommendations for low-dose-rate brachytherapy for carcinoma of the cervix. Int J Radiat Oncol Biol Phys 2002; 52: 33-48.

4. Corn B, Hanlon A, Pajak T et al. Technically accurate intracavitary insertions improve pelvic control and survival among patients with locally advanced carcinoma of the uterine cervix. Gynecol Oncol 1994; 53: 294-300.

5. Eifel PJ, Moughan J, Erickson B et al. Patterns of radiotherapy practice for patients with carcinoma of the uterine cervix: a patterns of care study. Int J Radiat Oncol Biol Phys 2004; 60: 1144-1153.

6. Inoue T. Quality assurance of radiotherapy and its clinical assessment. Jap J Clin Oncol 2002; 32: 497-505.

7. National Comprehensive Cancer Network. Clinical Practice Guidelines in Oncology - v.1.2003 - Cervical Cancer; available at: http://www.nccn.org/physician_gls/index.html.

8. Lang S, Kirisits C, Dimopoulos J et al. Treatment planning for MRI assisted brachytherapy of gynecologic malignancies based on total dose constraints. Int J Radiat Oncol Biol Phys 2007; 69: 619-627.

9. van Dyk S, Byram D, Bernshaw D. Brachytherapy for cancer of the cervix: an Australian and New Zealand survey of current treatment techniques. J Med Imag Radiat Oncol 2009; 52: 588-597.

10. Bernshaw D, van Dyk S, Bernshaw D et al. Comparative study of LDR (Manchester System) and HDR image-guided conformal brachytherapy of cervical cancer: patterns of failure, late complications, and survival. Int J Radiat Oncol Biol Phys 2009; 74: 1529-1535.

11. Toita T, Kodaira T, Shinoda A et al. Patterns of radiotherapy practice for patients with cervical cancer (1999-2001): patterns of care study in Japan. Int J Radiat Oncol Biol Phys 2008; 70: 788-794.
12. Pearce A, Craighead P, Kay I et al. Brachytherapy for carcinoma of the cervix: a Canadian survey of practice patterns in a changing era. Radiother Oncol 2009; 91: 194-196.

13. Erickson B, Eifel P, Moughan J et al. Patterns of brachytherapy practice for patients with carcinoma of the cervix (19961999): a patterns of care study. Int J Radiat Oncol Biol Phys 2005; 63: 1083-1092.

14. Ogino I, Kitamura T, Okamoto $\mathrm{N}$ et al. Late rectal complication following high dose rate intracavitary brachytherapy in cancer of the cervix. Int J Radiat Oncol Biol Phys 1995; 31: 725-734.

15. MacLeod C, Fowler A, Dalrymple C et al. High-dose-rate brachytherapy in the management of carcinoma of the cervix: an 8-year Australian experience. Aust NZ J Obstet Gyaecol 1997; 37: 342-347.

16. Opfermann KJ, Wahlquist A, Watkins J et al. Impact of point A asymmetry on local control and survival for low dose-rate (LDR) brachytherapy in cervical cancer. J Contemp Brachtherapy 2012; 4: 3-7.

17. Nag S, Orton C, Young D et al. The American Brachytherapy Society survey of brachytherapy for carcinoma of the cervix in the United States. Gynecol Oncol 1999; 73: 111-118.

18. Board of the Faculty of Clinical Oncology, The Royal College of Radiologists. The Role and Development of Brachytherapy Services in United Kingdom; available at: http://www. rcr.ac.uk.

19. Eifel PJ, Moughan J, Owen J et al. Patterns of radiotherapy practice for patients with squamous carcinoma of the uterine cervix: patterns of care study. Int J Radiat Oncol Biol Phys 1999; 43: 351-358.

20. Teshima T, Abe M, Ikeda H et al. patterns of care study of radiation therapy for cervix cancer in Japan: the influence of the stratification of institution on the process. Jap J Clin Oncol 1998; 28: 388-395.

21. Downing A, Mikeljevic JS, Haward B et al. Variation in the treatment of cervical cancer patients and the effect of consultant workload on survival: a population-based study. Eur J Cancer 2007; 43: 363-370.

22. Palmer AL, Bidmead M, Nisbet A. A survey of quality control practices for high dose rate (HDR) and pulsed dose rate (PDR) brachytherapy in the United Kingdom. J Contemp Brachytherapy 2012; 4: 232-240.

23. Dimopoulos JCA, De Vos V, Berger D et al. Inter-observer comparison of target delineation for MRI-assisted cervical cancer brachytherapy: application of the GYN GEC-ESTRO recommendations. Radiother Oncol 2009; 91: 166-172.

24. Perez CA, Grigsby PW, Chao C et al. Tumor size, irradiation dose, and long-term outcome of carcinoma of uterine cervix. Int J Radiat Oncol Biol Phys 1998; 41: 307-317.

25. Petereit DG, Sarkaria JN, Chappell R et al. The adverse effect of treatment prolongation in cervical cancer. Int J Radiat Oncol Biol Phys 1995; 32: 1301-1307. 